\title{
Catalytically Active Nanomaterials: Artificial Enzymes of Next Generation
}

\author{
Sanjay Singh* \\ Division of Biological and Life Sciences, School of Arts and Sciences, Ahmedabad University, Central Campus, Navrangpura, Ahmedabad \\ 380009, Gujarat, India
}

Received: November 17, 2017; Accepted: December 5, 2017; Published: December 10, 2017

*Corresponding author: Sanjay Singh, Division of Biological and Life Sciences, School of Arts and Sciences, Ahmedabad University, Central Campus, Navrangpura, Ahmedabad - 380009, Gujarat, India. Tel: +91-79-61911270. E-mail: sanjay.singh@ahduni.edu.in

\begin{abstract}
Due to the inherent limitations associated with natural enzymes, discovery and development of nanomaterials-based artificial enzymes (nanozymes) are highly desired. These nanozymes may address the issues with practical applications of natural enzymes such as high cost of synthesis, purification, storage and limited spectrum of catalytic activity. In this review article, a crisp description of the recent progress in exploring, bio catalytic activity and constructing nanozymes, including AuNPs, carbon-based nanomaterials, and $\mathrm{CeO}_{2}$ NPs are discussed. A brief description of new or enhanced applications of these nanozymes in bio diagnosis and therapeutics has also been provided. Additionally, a section is dedicated to the use of efficient modulators of nanozymes to improve the catalytic performance. Nanozymes exhibiting multi-enzyme activity such as core-shell of $\mathrm{Au}$ and $\mathrm{CeO}_{2} \mathrm{NPs}$, carbon-based nanomaterials, and $\mathrm{Au}$ and $\mathrm{SiO}_{2} \mathrm{NPs}$ is also discussed in detail. Based on the recent investigations reported by our group and others, it is expected that nanozymes containing unique and tunable properties and functions will attract tremendous research interest and generate novel opportunities in several avenues of biomedicines.
\end{abstract}

Keywords: Gold Nanoparticles; Cerium oxide; Peroxidase activity; Oxidase activity; Superoxide dismutase activity; Nanozymes

\section{Introduction}

Natural enzymes are extremely specific and efficient biocatalysts, facilitating almost every biochemical process in living organisms with rapid rate $\left(10^{19}\right.$ times) of reaction [1] Although the location and function of natural enzymes are well orchestrated, their practical utility is often compromised by some of their intrinsic hitches. These drawbacks could be the high cost of production, purification and storage, lower stability in extreme physiological conditions, lower recyclability, and the stringent requirement for optimum catalytic activity [2-4]. In this context, next-generation artificial enzymes are needed. Recent developments in nanotechnology have led to the generation of nanomaterials exhibiting the catalytic activities analogous to natural enzymes [5-10]. These catalytically active nanoparticles (nanozymes) seem to solve the issues related to natural enzymes, as they offer low cost of production, size, and shape mediated control over catalytic activity and high stability in extreme physiological conditions. Utilizing the enzymatic properties of these nanozymes, several detection methods of analytes in extremely low concentration has also been devised [7, 11-14]. Considering the above-mentioned advantages of nanozymes, in this review article, we comprehensively discuss the recent developments and future direction of nanomaterials as artificial enzymes.

Recent utilization of enzymes in the construction of biosensors, food industry, technological applications and agricultural research has seen prompt progress. Although several natural enzyme-based methods have been devised in these areas, however, the realization of actual potential has been not received due to the limitations associated with enzymes. In this context, merging nanotechnology with enzymology has paved a way to design novel multifunctional nanozymes exhibiting intrinsic biological enzyme-like activities. So far several types of nanomaterials such as gold $(\mathrm{Au})$, Silver $(\mathrm{Ag})$, Copper $(\mathrm{Cu})$ nanoparticles (NPs), Cerium oxide $\left(\mathrm{CeO}_{2}\right)$, iron oxide, $\mathrm{V}_{2} \mathrm{O}_{5}, \mathrm{Pt}, \mathrm{Pd}$, graphene, fullerenes, and carbon nano tubes etc [7,15-24]. have been investigated to possess the natural enzyme-like activities. Oxidase, peroxidase, superoxide dismutase (SOD) and catalase are some of the most studied enzymes for which nanomaterialsbased artificial enzymes have been investigated (Figure 1). Despite the vast variety of nanomaterials exhibiting enzyme-like activities, this review will only focus on the nanozymatic activity of gold nanoparticles. Since during last two decades, $\mathrm{CeO}_{2} \mathrm{NPs}$ and certain carbon based materials have emerged as potential antioxidants which have shown tremendous applications in treating oxidative stress based human diseases, towards the end of this review article, a short report will be provided on $\mathrm{CeO}_{2} \mathrm{NPs}$ and carbon-based nanomaterials.

Among metallic nanomaterials, AuNPs have been well explored for their enzyme-like activities. Such investigation becomes more intense as AuNPs offers high biocompatibility to 


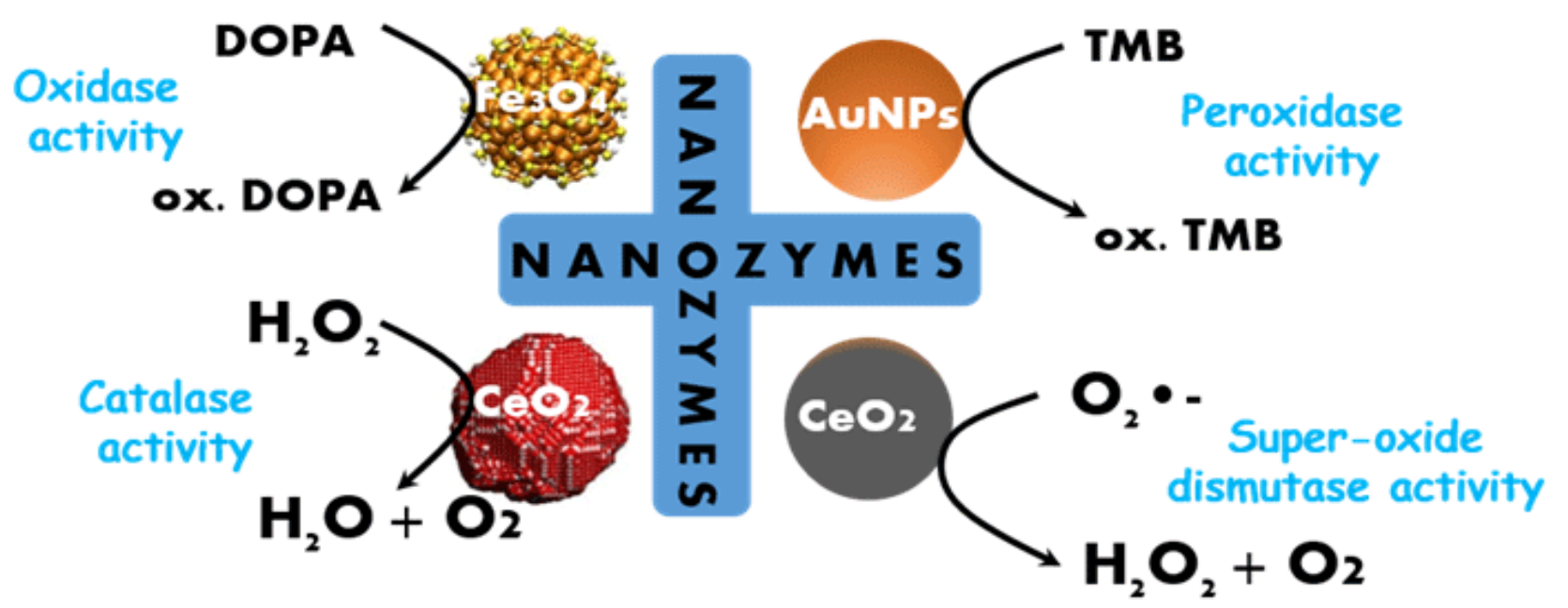

Figure 1: Schematic diagram showing oxidase, peroxidase, catalase and superoxide dismutase enzyme-like activities by $\mathrm{Fe}_{3} \mathrm{O}_{4}, \mathrm{AuNPs}$, and $\mathrm{CeO}_{2}$ nanoparticles, respectively. Dihydroxyphenylalanine (DOPA) and TMB are respectively oxidized during oxidase and peroxidase activity. However, during catalase activity, hydrogen peroxide is degraded into $\mathrm{H}_{2} \mathrm{O}$ and $\mathrm{O}_{2}$. Similarly, during superoxide dismutase activity, superoxide radicals are reduced into $\mathrm{H}_{2} \mathrm{O}_{2}$ and $\mathrm{O}_{2}$.

mammalian cells [25,26]. Although AuNPs have been considered as chemically inert materials, however, recent investigations reveal that AuNPs exhibit excellent peroxidase-like activity [27]. Mimicking the glucose oxidase-like activity of AuNPs has led researchers to prepare different types of glucose and other sensors [28]. Paper chip based simple, portable and cost-effective sensing platform has been developed for naked-eye detection of mercury. It was shown that multiple tests can be simultaneously executed on a single device and the result can easily be read by a camera of the smart phone. The limit of detection of this method was found to be $0.006 \mathrm{ng}$ for a single application and $0.012 \mathrm{ng}$ for five test sample analysis [29]. Recently Yang, et al. have developed gold nano rods (AuNRds) based monitoring of telomerase activity, which is a valuable marker for cancer diagnosis [30]. This strategy was a simple, naked eye based detection and label free method for telomerase sensing through enzymatic etching of AuNRds. The sensor contained repeating sequences (TTAGGG) extended on telomerase substrate primer where it formed a G-quadruplex in presence of Hemin and $\mathrm{K}+$ ions. Hemin/Gquadruplex exhibiting peroxidase-like activity catalyzes the $\mathrm{H}_{2} \mathrm{O}_{2}$ mediated etching of AuNRds into shorter rods and even to spherical AuNPs. Since, AuNRds exhibit size-dependent SPR and suspension color; it offers a visual detection of telomerase. Thus, this enzymatic reaction can be easily coupled to telomerase activity, allowing for the detection of telomerase activity based on vivid colors. Although several such bio sensing and other applications have been reported utilizing the peroxidase-like activity of AuNPs, the full potential of AuNPs as enzyme-mimics is still in infancy due to the low intrinsic catalytic activities and stability in biologically relevant buffers [31]. In this context, the emergence and recent advances of nanotechnology need to develop new strategies to develop nanomaterials or modification in sensing methods which could produce stable materials with high catalytic activity. Such developments would offer sensitive and early stage detection of biological analytes, which will be of tremendous help in developing nano medicines. With this in mind, several investigators have developed methods involving AuNPs, which can improve the present methodology. Among them, Lin et al. have reported a self-activated enzyme-mimetic system by using expanded mesoporous silica-encapsulated AuNPs, which exhibited two enzymes-like activity, glucose oxidase, and peroxidase activity. Mechanistically, mesoporous silica helps in the formation of a very small and well-dispersed AuNPs, which exhibit an extraordinary stability and dual enzyme-like activities [32]. These unique properties were further complexed together into a self-organized artificial cascade reaction involving the oxidase-peroxidase coupled enzyme system. Additionally, single component AuNPs are also expected not to show dual enzymelike functionalities, as unsupported AuNPs can keep only one type of enzyme activity while the other can be completely blocked [33, 34]. In this context, Singh and co-workers have developed $\mathrm{Au}$-core- $\mathrm{CeO}_{2}$-shell nanoparticles, which can show triple enzyme activity, peroxidase, catalase, and SOD [35,36]. It was interesting to observe that by the spatial arrangement of two materials as core-shell could lead to the three enzyme activities. Authors reported that, due to the redox behaviour at the interface of $\mathrm{Au}$ and $\mathrm{CeO}_{2}$, the third enzyme activity can be achieved. Apart from providing a support system to AuNPs which offers stability, other strategies are also reported where the peroxidase reaction products are stabilized. One such effort was reported by Shah et al [37]. where ATP (Adenosine triphosphate) was shown to enhance the peroxidase-like activity of citrate-stabilized AuNPs. Different sized of AuNPs (15, 30, 50 and $70 \mathrm{~nm}$ ) were studied, of which $30 \mathrm{~nm}$ exhibited synergistic peroxidase-like activity in presence 
of ATP. Further, the catalytic activity was found unaltered when the reaction was performed in presence of ADP, free phosphate, sulphate and carbonate anions, however, addition of ascorbic acid diminished the intrinsic peroxidase-like activity, even in the presence of ATP. A systematic study by authors revealed that presence of ATP did not enhance the production of hydroxyl radicals, which is central to the peroxidase-like activity exhibited by most of the nano materials, rather it diminishes the radical production in the reaction mixture. When investigated further, it was observed that ATP provides stability of oxidized TMB (3,3,5,5-tetramethylbenzidine), which is the reaction product of peroxidase activity. The biosensors developed on the concept of peroxidase-like activity read the absorbance at $650 \mathrm{~nm}$ of oxidized TMB (blue color). Very frequently it has been observed that the oxidized TMB undergoes auto-reduction and soon converts into reduced TMB, which leads to the loss of absorbance at $650 \mathrm{~nm}$ and concomitant loss of visual color. Such events limit the practical utility of peroxidase-like activity exhibited by nanomaterials. However, with the use of ATP, it was found that the oxidized TMB can be stabilized for extended hours, thus preserves the absorbance and blue visual color. On the similar concept, Lin et al. have demonstrated that with the addition of ionic liquid, $\mathrm{Au} / \mathrm{SiO}_{2}$ hetero-nano composites serve as a robust and recyclable peroxidase mimic even at high-temperature catalytic reactions [38]. Authors found that ionic liquid serves as a stabilizing agent and improved thermal stability of ABTS •+ radicals and subsequently enabled to facilitate the peroxidase reaction at high temperature, which was not possible in buffer solution.

Similar to ionic liquids and ATP, other nucleoside triphosphates (NTPs) are also reported to positively modulate the enzyme-like activity exhibited by nanomaterials, thus could serve asan enhanced activity when used with NTPs. Among used NTPs, UTP (Uridine triphosphate) and ATP showed improved oxidase enzyme-like activity, whereas UTP and CTP did not show any significant improvement in catalytic activity [39]. It was suggested that this effect is due to the coupling of the oxidative reaction with NTP hydrolysis. Different NTPs reflected different dephosphorylation activity by $\mathrm{CeO}_{2} \mathrm{NPs}$, which was further developed as an effective and high-throughput colorimetric assay for testing single-nucleotide polymorphism. Additionally, Singh et al. have studied the alteration in catalytic properties of $\mathrm{CeO}_{2}$ NPs when exposed to biologically relevant buffers [40, 41]. It was reported that phosphate anions significantly diminished the SOD activity of $\mathrm{CeO}_{2}$ NPs. Mechanistically, the electrostatic repulsion between cationic $\mathrm{CeO}_{2}$ are shielded by anionic phosphate ions, thus agglomeration of NPs occurs in form of cerium phosphate. Cerium phosphate formation blocks the redox cycling between $\mathrm{Ce}^{+3} / \mathrm{Ce}^{+4}$, and stabilizes the "Ce" in +3 oxidation state form. Considering the abundance of phosphate anions and related bio molecules in biological systems, it is likely that the action of $\mathrm{CeO}_{2}$ NPs as a catalyst in the biological system may be strongly influenced by the local concentration of phosphate in the cells/ tissues in which it has been introduced.

Similar to AuNPs and $\mathrm{CeO}_{2}$ NPs, carbon-based materials, such as graphene oxide, graphene-based nano composites, carbon nano tubes (CNTs), and carbon nano dots, have also been discovered to exhibit peroxidase and SOD enzyme-like activities [42-47]. Soluble derivatives of fullerenes have found protecting rats from iron dependent lipid peroxidation by co-infusion of carboxyfullerenes [48]. ROS (Reactive Oxygen Species) are well known to play a significant role in ischemia-reperfusion (stroke) injury, thus, using this as an experimental model, Lai et al reported that administration of fullerenes to ischemia animal model leads to the substantial reduction in lung injury [49].Use of the peroxidase-like activity of CNTs is reported to develop a label-free colorimetric detection system for single nucleotide polymorphism in human DNA [45]. Additionally, a composite of magnetic silica nanoparticles with CNTs are also developed as a unique strategy for selective detection of copper ions by click chemistry [46]. Owing to the exceptional emission properties, carbon nano dots are being looked as a potential replacement of quantum dots as a biocompatible imaging agent for biological systems. Wang et al have recently showed that carbon dots also exhibit high peroxidase-like activity, which can be used for sensitive and colorimetric detection of glucose from the real samples[50].

\section{Limitations and Future opportunities}

AuNPs, carbon, and $\mathrm{CeO}_{2}$ NPs based artificial nanozymes are less vulnerable to denaturation, low-cost, easy to synthesize, long shelf-life and are more stable than natural enzymes. Additionally, these nanozymes also exhibit unique features such as large surface area and shape and size-dependent properties, which can be used to tune the catalytic activity. Contrary to this, it is also essential to discuss the potential challenges limiting the full potential use of nanozymes in biomedicines as biocatalysts. Low catalytic activity is one of the biggest limitations with nanozymes. Therefore, more efforts need to be devoted to developing strategies to significantly improve the catalytic activity of nanozymes. Our group has recently started to address this issue by exploring the efficient boosting agents such as ATP. We have recently shown that with the use of ATP, the peroxidase activity of iron oxide NPs can be tuned to neutral $\mathrm{pH}$, which opens the opportunities for a myriad of biological applications [51]. Compared to natural enzymes, a lower binding affinity of nanozymes with the substrate is another issue, which needs to be addressed. Contrary to this, natural enzymes exhibit high binding affinity and substrate specificity due to the presence of unique catalytic microenvironment (active sites). Nanozymes could also be surface modified with functional groups similar to the groups present in the active sites of natural enzymes, thus enhanced binding affinity and substrate specificity. In this context, the surface chemistry of AuNPs, carbon-based materials, and $\mathrm{CeO}_{2}$ NPs are well characterized, which helps in modifying the surface of these materials with desired functional groups [4, 52]. A careful assessment of nanozymes catalytic reactions, it can be concluded that only redox-active nanomaterials can catalyze the biological reactions, which are hydrolytic reactions. However, natural enzymes can catalyze various types of biochemical reactions. Thus, significant efforts must be devoted to constructing novel nanozymes which 
can catalyze other types of biochemical reactions. The biggest challenge with nanozymes is the rational design, which can be solved by nanotechnology field itself $[53,54]$. Although the abovementioned issues are the key limitations which need immediate focus, however, future research in nanozymes technology is expected to grow with special emphasis to the exploitation of their potential applications in bio sensing and disease diagnosis and this information can be supported together into organized functional systems.

\section{Conclusions}

In last two decades, there have been several efforts by chemists and biologists to create synthetic structures that can mimic the functions of natural enzymes. In this context, nanomaterials are the most experimented materials and currently established as one of the promising alternatives to the natural enzymes. Among nanomaterials, AuNPs, carbon-based materials, and $\mathrm{CeO}_{2} \mathrm{NPs}$ are established as effective nanozymes exhibiting the properties of natural peroxidase, oxidase, catalase, and superoxide dismutase enzymes. The enzymatic activities of these biocompatible materials are successfully shown in several cell culture and animal models. This review report is an attempt to understand the mechanism and apply these nanomaterials as an artificial enzyme of the biological system and shed light on the strategies on improving the activities of these catalytic nanomaterials and their promising applications in detection, medical diagnosis, and therapeutics. Although it is well accepted that at present nanozymes face several unresolved issues and challenges, the intrinsic unique properties and tunable functions would lead to promising results and extraordinary outcomes as this field continues to thrive and mature in near future.

\section{Acknowledgement}

The financial assistance for the Centre for Nanotechnology Research and Applications (CENTRA) by the Gujarat Institute for Chemical Technology (GICT), Gandhi nagar and the funding from the Science and Engineering Research Board (SERB), India, Grant No. ILS/SERB/2015-2016/01 to Dr Sanjay Singh under the scheme of start-up research grant in Life Sciences is also acknowledged.

\section{References}

1. Lin Y, Ren J, Qu X. Catalytically Active Nanomaterials: A Promising Candidate for Artificial Enzymes. Accounts of chemical research. 2014;47:1097-1105.

2. Gao L, Zhuang J, Nie L, Zhang J, Zhang Y, Gu N, et al. Intrinsic Peroxidase-Like Activity of Ferromagnetic Nanoparticles. 2007;2(9):577-583. Doi: 10.1038/nnano.2007.260

3. Breslow R. Biomimetic control of chemical selectivity. 1980;13(6):170177. Doi:10.1021/ar50150a002

4. Kotov NA. Chemistry. Inorganic nanoparticles as protein mimics. 2010;330(6001):188-189. Doi:10.1126/science.1190094

5. Murakami Y, Kikuchi J, Hisaeda Y, Hayashida O. Artificial Enzymes. Chemical reviews. 1996;96(2):721-758. Doi: 10.1021/cr9403704

6. Singh V, Singh S, Das S, KumarA, Self WT, Seal S. A facile synthesis of PLGA encapsulated cerium oxide nanoparticles: release kinetics and biological activity. Nanoscale. 2012;4:2597-2605. Doi:10.1039/ C2NR12131J

7. Singh S. Cerium oxide based nanozymes: Redox phenomenon at biointerfaces. Biointerphases. 2016;11(4):04B202. Doi: 10.1116/1.4966535

8. Karakoti A, Singh S, Dowding JM, Sealacd S, Self WT. Redox-active radical scavenging nanomaterials. Chemical Society reviews. 2010;39(11):4422-4432.

9. Reema S,Darshini S,Ragini S, Ashutosh K, Rishi S, Alok D, et al. Nanotechnology in Disease Diagnostic Techniques. Current drug metabolism. 2015;16(8):645-661.

10. Liang M, Fan K, Pan Y, Jiang H, Wang F, Yang D, et al. Fe304 Magnetic Nanoparticle Peroxidase Mimetic-Based Colorimetric Assay for the Rapid Detection of Organophosphorus Pesticide and Nerve Agent. Analytical chemistry.2013;85(1):308-312. Doi: 10.1021/ac302781r

11. Ma Y, Zhang Z, Ren C, Liuab G, Chen X. A novel colorimetric determination of reduced glutathione in A549 cells based on Fe304 magnetic nanoparticles as peroxidase mimetics. The Analyst.2012;137:485-489.

12.Ni P, Dai H, Wang Y,Sun Y,Shi Y, Hu J, Lia Z. Visual detection of melamine based on the peroxidase-like activity enhancement of bare gold nanoparticles. Biosensors \& bioelectronics. 2014;60:286291.

13. Maji SK, Mandal AK, Nguyen KT, Borah P, Zhao Y. Cancer Cell Detection and Therapeutics Using Peroxidase-Active Nanohybrid of Gold Nanoparticle-Loaded Mesoporous Silica-Coated Graphene. ACS applied materials \& interfaces. 2015;7(18):9807-9816. Doi: 10.1021/acsami.5b01758

14. Sharma TK, Ramanathan R, Weerathunge P, Mohammadtaheri M, Daima HK, Shuklaa R,et al. Aptamer-mediated 'turn-off/turn-on' nanozyme activity of gold nanoparticles for kanamycin detection. Chemical communications (Cambridge, England).2014; 50:1585615859. Doi:10.1039/C4CC07275H

15. Lien CW, Huang CC, Chang HT. Peroxidase-mimic bismuthgold nanoparticles for determining the activity of thrombin and drug screening. Chemical communications (Cambridge, England).2012;48:7952-7954. Doi:10.1039/C2CC32833J

16. Jiang H, Chen Z, Cao H, Huang Y. Peroxidase-like activity of chitosan stabilized silver nanoparticles for visual and colorimetric detection of glucose. The Analyst, 2012;137:5560-5564. Doi:10.1039/C2AN35911A

17. Chen W, Chen J, Feng YB, Hong L, Chen QY, Wu LF,et al. Peroxidase-like activity of water-soluble cupric oxide nanoparticles and its analytical application for detection of hydrogen peroxide and glucose. The Analyst. 2012;137:1706-1712. Doi:10.1039/C2AN35072F

18. Yu F, Huang Y, Cole AJ, Yang VC. The artificial peroxidase activity of magnetic iron oxide nanoparticles and its application to glucose detection. Biomaterials. 2009;30:4716-4722.

19. André $R$, Natálio $F$, Humanes $M$, Leppin J, Heinze $K$, Weve R. V205 Nanowires with an Intrinsic Peroxidase-Like Activity. Advanced Functional Materials. 2010;21(3):501-509. Doi: 10.1002/ adfm. 201001302

20.Ju Y, Kim J. Chemical communications (Cambridge, England).2015;51:13752-13755. 
21. Wei J, Chen X, Shi S, Mo S, Zheng N. An investigation of the mimetic enzyme activity of two-dimensional Pd-based nanostructures. Nanoscale. 2015;7:19018-19026.

22. Sun R, Wang Y, Ni Y, Kokot S. Spectrophotometric analysis of phenols, which involves a hemin-graphene hybrid nanoparticles with peroxidase-like activity. Journal of hazardous materials. 2014;266:6067.

23. Li R, Zhen M, Guan M, Chen D, Zhang G, Ge J. A novel glucose colorimetric sensor based on intrinsic peroxidase-like activity of C60carboxyfullerenes. Biosensors \& bioelectronics. 2013;47:502-507.

24. Wang T, Fu Y, Chai L, Chao L, Bu L, Meng Y, et al. Chemistry (Weinheim an der Bergstrasse, Germany).2014;20:2623-2630.

25. Singh S. Glucose decorated gold nanoclusters: A membrane potential independent fluorescence probe for rapid identification of cancer cells expressing Glut receptors. Colloids and Surfaces B: Biointerfaces. 2017;155:25-34.

26. Singh S, 'Britto V D, Prabhune A A, Ramana CV , Dhawan A, Prasad BLV . Cytotoxic and genotoxic assessment of glycolipid-reduced and -capped gold and silver nanoparticles. New Journal of Chemistry. 2010;34:294-301. Doi:10.1039/B9NJ00277D

27. Comotti M, Pina CD, Matarrese R, Rossi M. The Catalytic Activity of "Naked" Gold Particles. AngewandteChemie International ed. 2004;43:5812-5815. Doi: 10.1002/anie.200460446

28. Lin Y, Ren J, Qu X . Nano-gold as artificial enzymes: hidden talents. Advanced materials (Deerfield Beach) Fla. 2014;26(25):4200-4217. Doi: 10.1002/adma.201400238

29. Han KN, Choi JS, Kwon J. Gold nanozyme-based paper chip for colorimetric detection of mercury ions. Scientific reports. 2017;7:2806. Doi: 10.1038/s41598-017-02948-x

30. Yang H, Liu A, Wei M, Liu Y, Lv B, Wei W. Visual, Label-Free Telomerase Activity Monitor via Enzymatic Etching of Gold Nanorods. Analytical chemistry. 2017;89 (22):12094-12100. Doi: 10.1021/acs. analchem.7b02608

31. Zheng X, Liu Q, Jing C, Li Y, Li D, Luo W, et al. AngewandteChemie (International ed). 2011;50:11994-11998.

32. Lin Y, Li Z,Chen Z, Ren J, Qu X. Mesoporous silica-encapsulated gold nanoparticles as artificial enzymes for self-activated cascade catalysis. Biomaterials. 2013;34:2600-2610.

33. Luo W, Zhu CF, Su S, Li D, He Y, Huang Q. Self-Catalyzed, Self-Limiting Growth of Glucose Oxidase-Mimicking Gold Nanoparticles. ACS nano. 2010;4(12):7451-7458.

34. Yun JV, Baoxin L, Caoa R. Positively-charged gold nanoparticles as peroxidiase mimic and their application in hydrogen peroxide and glucose detection.Chemical communications (Cambridge, England). 2010;46:8017-8019. Doi:10.1039/C0CC02698K

35. Bhagat S, Vallabani NV, Shutthanandan V, Bowden M, Karakoti AS, S. S. Journal of colloid and interface science. 2018.

36. Bhagata S, Srikanth NV, Shutthanandanb V, Bowdenb M, Karakotia AS, Singha S. Gold core/Ceria shell-based redox active nanozyme mimicking the biological multienzyme complex phenomenon. Journal of colloid and interface science, 2017.

37. Shah J, Purohit R, Singh R, Singh AKi, Singh S. ATP-enhanced peroxidase-like activity of gold nanoparticles. Journal of colloid and interface science. 2015;456:100-107.
38. Lin Y, Zhao A, Tao Y, Ren J, Qu X. Ionic Liquid as an Efficient Modulator on Artificial Enzyme System: Toward the Realization of HighTemperature Catalytic Reactions. Journal of the American Chemical Society.2013;135(11):4207-4210.

39. Asati A, Santra S, Kaittanis C, Nath S, Perez JM. Oxidase-like activity of polymer-coated cerium oxide nanoparticles. AngewandteChemie (International ed). 2009;48:2308-2312.

40.Singh S, Dosani T, Singh AKi, Kumar A, Seal S, Selfa WT. A phosphatedependent shift in redox state of cerium oxide nanoparticles and its effects on catalytic properties. Biomaterials. 2011;32:6745-6753.

41. Singh R, Singh S. Role of phosphate on stability and catalase mimetic activity of cerium oxide nanoparticles. Colloids and surfaces. 2015;132:78-84.

42. Song Y, Qu K, Zhao C, Ren J, Qu X. Graphene oxide: intrinsic peroxidase catalytic activity and its application to glucose detection. Advanced materials (Deerfield Beach) Fla. 2010;22:2206-2210. Doi: 10.1002/ adma. 200903783

43.Yu Tao, Youhui Lin, Jinsong Ren, Xiaogang Qu. Self-assembled, functionalized graphene and DNA as a universal platform for colorimetric assaysBiomaterials. 2013;34(20):4810-4817.

44. Song Y, Chen Y, Feng L, Rena J, Qu X. Selective and quantitative cancer cell detection using target-directed functionalized graphene and its synergetic peroxidase-like activity. Chemical communications (Cambridge, England).2011;47:4436-4438.

45. Song Y, Wang X, Zhao C, Qu K, Ren J, Qu X. Chemistry (Weinheim an der Bergstrasse, Germany). 2010;16:3617-3621.

46.Song Y, Qu K, Xu C, Ren J, Qu X. Chemical communications (Cambridge, England). 2010;46:6572-6574.

47. Das S, Singh S, Singh V, Joung D, Dowding JM, Reid D, et al. Oxygenated Functional Group Density on Graphene 0xide: Its Effect on Cell Toxicity. Particle \& Particle Systems Characterization.2013;30:148-157. Doi: 10.1002/ppsc.201200066

48. Lin AMY, Chyi BY, Wang SD, Yu HH, Kanakamma PP, Luh TY, et al. Carboxyfullerene Prevents Iron-Induced Oxidative Stress in Rat Brain. Journal of neurochemistry. 1999;72:1634-1640. Doi: 10.1046/j.14714159.1999.721634.x

49. Lai YL, Murugan P, Hwang KC. Fullerene derivative attenuates ischemia-reperfusion-induced lung injury. Life sciences. 2003;72: 1271-1278.

50. Wang X, Qu K, Xu B, Ren J, Qu X. Multicolor luminescent carbon nanoparticles: Synthesis, supramolecular assembly with porphyrin, intrinsic peroxidase-like catalytic activity and applications. Nano Research. 2011;4(9):908-920.

51. Srikanth NV, Singh AK, Singh S. ATP-mediated intrinsic peroxidaselike activity of Fe304-based nanozyme: One step detection of blood glucose at physiological pH Colloids and surfaces. 2017;153:52-60.

52. Singh AK, Shukla R, Shanker R, Singh S. Surface functionalization of quantum dots for biological applications. Advances in colloid and interface science. 2015;215:28-45.

53. Valden M, Lai X, Goodman DW. Onset of catalytic activity of gold clusters on titania with the appearance of nonmetallic properties. 1998;281:1647-1650.

54.Yamada Y, Tsung CK, Huang W, Huo Z, Habas SE, Soejima $\mathrm{T}$, et al. Nanocrystal bilayer for tandem catalysis. Nature chemistry.2011;3:372-376. Doi:10.1038/nchem.1018 